\title{
Asymptomatic SARS-CoV-2 seropositivity: patients with childhood-onset rheumatic diseases versus healthy children
}

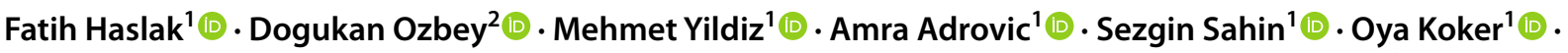

 \\ Ozgur Kasapcopur $^{1}$ (1) $\cdot$ Kenan Barut $^{1}$ (1)
}

Received: 17 June 2021 / Revised: 10 January 2022 / Accepted: 13 January 2022 / Published online: 19 January 2022

(c) International League of Associations for Rheumatology (ILAR) 2022

\begin{abstract}
Objective We aimed to find out the asymptomatic severe acute respiratory syndrome coronavirus 2 (SARS-CoV-2) seroprevalence among pediatric patients with rheumatic diseases and healthy children and to compare them with each other.

Methods Patients with familial Mediterranean fever (FMF), juvenile idiopathic arthritis (JIA), and juvenile systemic lupus erythematosus (jSLE) and healthy children as healthy control (HC) group who remained asymptomatic during the pandemic are examined by ELISA immunoglobulin (Ig) A and IgG tests in this cross-sectional study.

Results Overall, 149 subjects (90 females) were included in the study. While IgA was positive in 15 subjects (10\%) (HC: 8, jSLE: 3, FMF: 2, JIA: 2; $p=0.196)$, IgG was positive in 14 subjects (9.4\%) (HC: 7, JIA: 5, FMF: 1 , jSLE: $1 ; p=0.156)$. Nineteen subjects (12.75\%) were IgA or IgG positive (HC: 8, JIA: 5, jSLE: 3, FMF: 3; $p=0.644$ ). Although not significant, seropositivity was more often in $\mathrm{HC}$ group. Both $\operatorname{IgA}$ and $\mathrm{IgG}$ positivity were not found to be related to age, sex, underlying rheumatic diseases, and received treatments of the patients.

Conclusion We revealed that patients with childhood-onset rheumatic diseases, even if they receive immunosuppressive medication such as biologic or conventional disease-modifying anti-rheumatic drugs, might have an asymptomatic SARSCoV-2 infection, similarly to their healthy peers.

\section{Key points}

- Although it has been already known that children are most likely to have asymptomatic SARS-CoV-2 infection, there is a lack of data on the disease course of children with rheumatic disease.

- There was no significant difference regarding the asymptomatic SARS-CoV-2 seropositivity rates between healthy children and the patients with childhood-onset rheumatic diseases.

- Patients with childhood-onset rheumatic diseases, even if they receive immunosuppressive medication, might have asymptomatic SARS-

CoV-2 infection, similarly to their healthy peers.
\end{abstract}

Keywords COVID-19 · Familial Mediterranean fever $\cdot$ Juvenile idiopathic arthritis $\cdot$ SARS-CoV-2 $\cdot$ Systemic lupus erythematosus

\section{Introduction}

Coronavirus disease 2019 (COVID-19), caused by a novel coronavirus named severe acute respiratory syndrome coronavirus 2 (SARS-CoV-2), emerged in late December 2019. Due to the rapidly growing number of cases, the World

Kenan Barut

drkenanbarut@hotmail.com

Extended author information available on the last page of the article
Health Organization (WHO) declared a pandemic on March 11 th 2020 [1].

Although 10-20\% of the infected individuals experience life-threatening events, children are most likely to have a significantly milder disease course [2]. Raised concerns regarding the vulnerability of those with several comorbidities led to studies evaluating the patients with rheumatic diseases, but they were not found to be significant risk factors for a severe disease course, neither in childhood nor in adulthood [3-6]. 
Data regarding clinical features of COVID-19 in children are limited compared to adults, which may be attributed to possibly higher rates of asymptomatic cases among children [7]. Even if the SARS-CoV-2-infected children are most likely to remain asymptomatic, they might have a pivotal role in the transmission. In a recent study, the duration of viral shedding of SARS-CoV-2 from the respiratory tract and stool of symptomatic and asymptomatic children were compared and did not find a significant difference. Thus, the authors emphasized a challenge raised by asymptomatic children in disease control [8].

To minimize this challenge, a school lockdown during the pandemic is considered an effective preventive strategy by several countries. However, seroconversion frequencies of school attender children and the children who chose remote learning were compared in an Italian study and found to be similar. Therefore, it was suggested that the schools do not have a significant role in SARS-CoV-2 transmission [9].

Since the children are considered hidden drivers of the pandemic, an urgent need for studies evaluating the frequency of pediatric carriers, including those with underlying diseases, emerged. Therefore, we aimed to find out the asymptomatic SARS-CoV-2 seroprevalence among our pediatric patients with rheumatic diseases and healthy children and to compare them with each other.

\section{Materials and methods}

\section{Study design}

In our daily pediatric rheumatology practice, the most common diseases are familial Mediterranean fever (FMF), vasculitis, juvenile idiopathic arthritis (JIA), and juvenile systemic lupus erythematosus (jSLE). Therefore, to evaluate the asymptomatic SARS-CoV-2 seropositivity among children with rheumatic diseases, patients with FMF, JIA, and jSLE were selected as a patient group.

"Systemic Lupus International Collaborating Clinics" classification criteria [10], Turkish Pediatric FMF criteria [11], and "The International League of Associations for Rheumatology (ILAR)" criteria [12] were used to establish the diagnoses of jSLE, FMF, and JIA, respectively. Children who were previously admitted to our hospital due to a non-specific and transient complaint before the date of the first COVID-19 case were seen in our country and without any diagnosed underlying disease were established as the control group.

\section{Questionnaire}

We planned a cross-sectional study between August and October 2020, involving 180 subjects. A web-based survey was prepared and sent to the families in mid-August 2020. Contact history with a confirmed COVID-19 case and symptoms suggestive for COVID-19 during the pandemic (for the last 5 months) were questioned.

Considering that the 11th of March 2020 is the day of the declaration of a pandemic by the World Health Organization, which is also the day that the first COVID-19 case was seen in our country, to assess asymptomatic subjects, symptoms that appeared during the last 5 months were questioned. Each of the symptoms which are suggestive of COVID-19, such as high fever, cough, sore throat, rhinorrhea, headache, anosmia, rashes, and diarrhea, was asked, one by one.

Those who remained asymptomatic for the last 5 months were called via phone, and their answers were verified. Patients diagnosed with FMF, JIA, and jSLE before the age of 18 and currently being under 21 and healthy individuals under 18 are included in the study.

\section{Face-to-face appointments}

Among the participants whose answers were confirmed and who met the inclusion criteria, 180 subjects were randomized into FMF, JIA, jSLE, and healthy control (HC) groups in an equal number, regardless of their contact histories. They were called via phone, and a face-to-face appointment was arranged for each of them. During the face-to-face appointment, data regarding the COVID-19 were re-checked and verified for the second time in mid-September 2020.

\section{Blood sample collection and monitoring}

Blood samples were obtained from those who approved the study and those who still have no symptoms suggestive of the COVID-19. It was previously shown that the maximum incubation period of SARS-CoV-2 is 14 days [13]. Therefore, 2 weeks after the blood samples of the subjects were taken, the families were called via phone one by one, results were declared, symptoms were asked again, and those who had any symptoms suggestive for COVID-19 in this last 2-week period were excluded from the study.

Additionally, to detect post-infectious complications such as multi-inflammatory syndrome in children (MIS-C), the families of seropositive subjects were called via phone 1 month after, and 2 months afterward. 


\section{Final study population}

Our web-based survey was sent to the families of 1294 subjects, among whom 933 (72.1\%) responded back, and it was seen that 327 (25.2\%) had remained asymptomatic for the last 5 months. Due to the limited count of antibody commercial kits, 180 of 327 asymptomatic participants were randomized.

Among these randomized 180 subjects, 19 (10.5\%) developed symptoms suggestive of COVID-19 until their appointments, and one $(0.55 \%)$ within 2 weeks after the blood sample was taken. Seven (3.88\%) subjects did not attend their appointments, $3(1.66 \%)$ subjects did not approve the informed content, and blood samples of one $(0.55 \%)$ could not be taken due to agitation. Finally, 149 subjects were eligible for the study.

\section{Ethical approval}

The institutional ethics committee of our tertiary center approved the study protocol (04/16/2029,430,533-604.01-01-54,959). We followed the recommendations of the Declaration of Helsinki for biomedical research involving human subjects. Informed consent was obtained from the participants, and/or from their caregivers included in the study.

\section{Antibody testing}

Approximately $5 \mathrm{ml}$ of the whole blood samples was drawn from each subject to a yellow cap vacutainer and transferred to the microbiology/serology laboratory. The sera were separated from the whole blood samples by centrifugation at $4500 \mathrm{rpm}$ and stored at $-20^{\circ} \mathrm{C}$ until testing.

Detection of antibodies against SARS-CoV-2 spike protein from the serum sample of subjects was assayed by the ELISA method. Both immunoglobulin (Ig) A and IgG ELISA test kits (EUROIMMUN AG, Lübeck, Germany) are based on the sandwich and semi-quantitative principles. Assays were carried out according to the manufacturer's instructions with a dilution ratio of 1:100. Microplates were read by an automated microplate reader (BioTek ELx800, Istanbul, Turkey) at $450 \mathrm{~nm}$ (reference $620-650 \mathrm{~nm}$ ) wavelength, and absorbance (optical density, OD) of each serum sample was calculated.

The ratio of each sample was calculated by dividing the sample's OD by Calibrator's OD. A ratio higher than 1.1 is regarded as positive, and a ratio lower than 0.8 is regarded as negative. If the ratio was between 0.8 and 1.1, it is considered to be borderline (gray zone) according to the manufacturer's instructions. Results were calculated as semi-quantitative. Subjects with borderline ratios were regarded as negative or positive, depending on the expert opinion of the microbiologists.

\section{Statistical analysis}

The statistical analysis was performed using the IBM SPSS Statistics for Windows, Version 21.0 (Armonk, NY: IBM Corp). Normality was assessed with the Kolmogorov-Smirnov test. Continuous variables were presented as median and minimum-maximum or mean and standard deviation, based on their distributions. Categorical variables were compared using the chi-square test or, where appropriate, Fisher's exact test. A double-sided $p$-value of $\leq 0.05$ was considered statistically significant.

\section{Results}

\section{Study population}

Overall, 149 subjects (HC: $n=43$ (28.9\%); JIA: $n=42$ (28.2\%); jSLE: $n=33$ (22.1\%); FMF: $n=31(20.8 \%)$ ) were recruited to the study. The mean age was $12.86 \pm 4.76$ years, and $90(60.4 \%)$ of them were females. The subjects in the jSLE group were significantly older compared to others $(p<0.001)$. Additional demographic data are given in Table 1.

\section{Patients' group}

Fourteen patients (13.2\%) experienced underlying rheumatic disease-related symptoms during the pandemic (JIA: 9, FMF: 4, jSLE: 1); those with JIA presented with isolated arthritis, and those with FMF presented with fever and peritoneal irritation, which spontaneously resolved within 1-3 days and was considered to be a typical FMF attack by pediatric rheumatologists. Although one subject with jSLE presented with fever, chest pain, and pancytopenia, a polymerase chain reaction (PCR) test was performed and found negative. All the symptoms of these patients were attributed to their existing rheumatic condition. Thus, none of these subjects was considered symptomatic for COVID-19, and they were also included in the study.

Among those who experienced symptoms related to their rheumatic disease, only one FMF patient was seropositive (IgA: positive, IgG: negative). Those with FMF hydrated in their acute phase, and colchicine dosages were rearranged for all of them. Since all of those with JIA were steroid-free, $2 \mathrm{mg} / \mathrm{kg} /$ day methylprednisolone treatment with a tapering plan within 1 month was started for all. Biologic treatment, which she is currently receiving, was started in one of the JIA patients. One with jSLE was in clinical remission, and 
Table 1 Baseline characteristics of the study population

\begin{tabular}{|c|c|c|c|c|c|}
\hline & FMF group $(n=31)$ & JIA group $(n=42)$ & jSLE group $(n=33)$ & HC group $(n=43)$ & $p$ \\
\hline Age (years) $($ mean $\pm S D)$ & $12.95 \pm 4.17$ & $12.24 \pm 4.98$ & $16.18 \pm 4.07$ & $10.85 \pm 4.16$ & $<0.001$ \\
\hline Gender $(n, \%)$ & & & & & 0.869 \\
\hline Male & $13(41.9 \%)$ & $17(40.5 \%)$ & $11(33.3 \%)$ & $18(41.9 \%)$ & \\
\hline Female & $18(58.1 \%)$ & $25(59.5 \%)$ & $22(66.7 \%)$ & $25(58.1 \%)$ & \\
\hline Total household population (median, min-max) & $4(3-6)$ & $4(3-8)$ & $5(2-10)$ & $4(2-7)$ & 0.375 \\
\hline $\begin{array}{l}\leq 21 \text { years old household population (median, } \\
\text { min-max) }\end{array}$ & $2(1-3)$ & $2(1-6)$ & $1(1-6)$ & $2(1-4)$ & 0.497 \\
\hline $\begin{array}{l}\geq 65 \text { years old household population (median, } \\
\text { min-max) }\end{array}$ & $0(0-1)$ & $0(0-2)$ & $0(0-1)$ & $0(0-0)$ & 0.078 \\
\hline Contact history $(n, \%)$ & & & & & 0.046 \\
\hline Yes & $1(3.2 \%)$ & $0(0 \%)$ & $0(0 \%)$ & $4(9.3 \%)$ & \\
\hline No & $30(96.8 \%)$ & $42(100 \%)$ & $33(100 \%)$ & $39(91.7 \%)$ & \\
\hline Smoking exposure $(n, \%)$ & & & & & 0.018 \\
\hline Yes & $25(80.6 \%)$ & $25(59.5 \%)$ & $16(48.5 \%)$ & $20(46.5 \%)$ & \\
\hline No & $6(19.4 \%)$ & $17(40.5 \%)$ & $17(51.5 \%)$ & $23(53.5 \%)$ & \\
\hline Supplemental vitamin during the pandemic $(n, \%)$ & & & & & 0.071 \\
\hline Receiving & $11(35.5 \%)$ & $6(14.3 \%)$ & $4(12.1 \%)$ & $11(25.6 \%)$ & \\
\hline Not receiving & $20(64.5 \%)$ & $36(85.7 \%)$ & $29(87.9 \%)$ & $32(75.4 \%)$ & \\
\hline Treatment during pandemic $(n, \%)$ & & & & & - \\
\hline Steroid & $0(0 \%)$ & $9(21.4 \%)$ & $11(33.3 \%)$ & $0(0 \%)$ & \\
\hline NSAIDs & $1(3.2 \%)$ & $0(0 \%)$ & $1(3 \%)$ & $0(0 \%)$ & \\
\hline ASA & $0(0 \%)$ & $0(0 \%)$ & $5(15.2 \%)$ & $0(0 \%)$ & \\
\hline HCQ & $0(0 \%)$ & $0(0 \%)$ & $26(78.7 \%)$ & $0(0 \%)$ & \\
\hline Colchicine & $31(100 \%)$ & $0(0 \%)$ & $0(0 \%)$ & $0(0 \%)$ & \\
\hline bDMARDs & $0(0 \%)$ & $22(52.4 \%)$ & $0(0 \%)$ & $0(0 \%)$ & \\
\hline cDMARDs & $0(0 \%)$ & $23(54.8 \%)$ & $15(45.5 \%)$ & $0(0 \%)$ & \\
\hline None & $0(0 \%)$ & $7(16.6 \%)$ & $5(15.1 \%)$ & $43(100 \%)$ & \\
\hline IgA ratios (median, min-max) & $0.228(0.064-1.530)$ & $0.159(0.048-5.301)$ & $0.262(0.100-2.464)$ & $0.352(0.151-8.595)$ & - \\
\hline IgG ratios (median, min-max) & $0.201(0.105-2.233)$ & $0.219(0.096-5.099)$ & $0.183(0.080-5.164)$ & $0.289(0.156-9.644)$ & - \\
\hline Qualitative IgA (n, \%) & & & & & 0.196 \\
\hline Positive & $2(6.5 \%)$ & $2(4.8 \%)$ & $3(9.1 \%)$ & $8(18.6 \%)$ & \\
\hline Negative & $29(93.5 \%)$ & $40(95.2 \%)$ & $30(90.9 \%)$ & $35(81.4 \%)$ & \\
\hline Qualitative $\operatorname{Ig} G(n, \%)$ & & & & & 0.156 \\
\hline Positive & $1(3.2 \%)$ & $5(11.9 \%)$ & $1(3 \%)$ & $7(16.3 \%)$ & \\
\hline Negative & $30(96.8 \%)$ & $37(88.1 \%)$ & $32(97 \%)$ & $36(83.7 \%)$ & \\
\hline $\begin{array}{l}\text { *Do the parents think that rheumatic disease } \\
\text { increases the risk of SARS-CoV-2 infection? }\end{array}$ & & & & & 0.724 \\
\hline Yes & $26(83.9 \%)$ & $32(76.2 \%)$ & $26(78.8 \%)$ & - & \\
\hline No & $5(16.1 \%)$ & $10(23.8 \%)$ & $7(21.2 \%)$ & - & \\
\hline $\begin{array}{l}\text { *Underlying rheumatic disease-related symptoms } \\
(n, \%)\end{array}$ & & & & & 0.053 \\
\hline Experienced & $4(12.9 \%)$ & $9(21.4 \%)$ & $1(3 \%)$ & - & \\
\hline Not experienced & $27(87.1 \%)$ & $33(78.6 \%)$ & $32(97 \%)$ & - & \\
\hline *Drug compliance during the pandemic $(n, \%)$ & & & & & 0.914 \\
\hline Regular & $28(90.3 \%)$ & $39(92.9 \%)$ & $30(90.9 \%)$ & - & \\
\hline Poor & $3(9.7 \%)$ & $3(7.1 \%)$ & $3(9.1 \%)$ & - & \\
\hline *Regular follow-ups during the pandemic (n, \%) & & & & & 0.516 \\
\hline Attended & $7(22.6 \%)$ & $8(19 \%)$ & $10(30.3 \%)$ & - & \\
\hline Not attended & $24(77.4 \%)$ & $34(81 \%)$ & $23(69.7 \%)$ & - & \\
\hline
\end{tabular}

$A S A$, acetyl salicylic acid; $b D M A R D s$, biologic disease-modifying anti-rheumatic drugs; $c D M A R D s$, conventional disease-modifying anti-rheumatic drugs; $F M F$, familial Mediterranean fever; $H C$, healthy control; $H C Q$, hydroxychloroquine; JIA, juvenile idiopathic arthritis; $j S L E$, juvenile systemic lupus erythematosus; NSAIDs, non-steroid anti-inflammatory drugs; SARS-CoV-2, severe acute respiratory syndrome coronavirus 2 *The control group was not taken into account in the analyses of these variables 
not under any medication. Hydroxychloroquine (HCQ) was re-started, intravenous immunoglobulin with a dose of 1 $\mathrm{gr} / \mathrm{kg}$ was administered, and $2 \mathrm{mg} / \mathrm{kg} /$ day methylprednisolone was started. His steroid treatment was stopped after 2 months in a stepwise manner. Additional data are given in Table 1.

\section{Antibody tests}

While IgA was positive in 15 subjects (10\%) (HC: 8 , jSLE: 3, FMF: 2, JIA: 2 ; $p=0.196)$, IgG was positive in 14 subjects (9.4\%) (HC: 7, JIA: 5, FMF: 1, jSLE: $1 ; p=0.156$ ). Nineteen subjects $(12.75 \%)$ were IgA or IgG positive (HC: 8, JIA: 5, jSLE: 3, FMF: 3; $p=0.644$ ). Besides, MIS-C was developed in none of the seropositive subjects.

In ELISA tests, three borderline values were determined, two for IgA and one for IgG. One of the subjects whose IgA results were borderline (IgA ratio $=0.92$ and IgG ratio $=2.23$ ) was in the FMF group, while the other $(\operatorname{IgA}$ ratio $=1.00$ and $\mathrm{IgG}$ ratio $=1.89)$ was in the $\mathrm{HC}$ group. Due to positivity of the IgG in both, the IgA results have been considered positive, as well. Furthermore, a contact history with confirmed COVID-19 case in a patient from FMF patients' group supported the borderline value of IgA antibody to be accepted as positive. Another patient from $\mathrm{HC}$ group had a borderline $\mathrm{IgG}$ result while the $\mathrm{IgA}$ antibody was negative (IgA ratio $=0.35$ and $\operatorname{IgG}$ ratio $=0.82$ ). The negativity of the IgA was a reason to accept this patient as seronegative and borderline IgG level was considered a result of crossmatch reaction due to previous sessional coronavirus infections. All of the IgA and IgG ratios of the participants are given in Fig. 2.

The mean age of the $\operatorname{IgA}$ and $\operatorname{IgG}$ positive subjects was $11.62 \pm 5.11(p=0.391)$ and $11.18 \pm 5.95(p=0.433)$ years, respectively. Ten $(66.7 \%)$ of the IgA positive subjects $(p=0.807)$, and $9(64.3 \%)$ of the IgG positive subjects $(p=0.980)$ were female. Seven of the $\operatorname{IgA}(46.7 \%$, $p=0.523)$ and seven of the $\operatorname{IgG}$ positive $(50 \%, p=0.741)$ subjects had a history of smoking exposure. Three (20\%) of the $\operatorname{IgA}$ positive subjects $(p=1)$ and $4(28.6 \%)$ of the IgG positive subjects $(p=0.501)$ were receiving supplemental vitamins during the pandemic.

The median of the total household population, of those younger than 21 and those older than 65 , was 5 (3-6) $(p=0.258), 2(1-3)(p=0.761)$, and $0(0-0)(p=0.251)$ in IgA positive subjects, and 5 (3-6) $(p=0.535), 2(1-3)$ $(p=0.540)$, and $0(0-1)(p=0.954)$ in IgG positive subjects, respectively.

During the pandemic, $3(20 \%, p=1)$ of the $\operatorname{IgA}$ positive subjects were receiving HCQ, $2(13.3 \%, p=0.738)$ were receiving colchicine, one was receiving steroids $(6.7 \%, p=0.694)$, one was receiving conventional disease-modifying anti-rheumatic drugs (cDMARDs) (azathioprine: 1) $(6.7 \%, p=0.116)$, and one was receiving biologic DMARDs (bDMARDs) (adalimumab: 1) (6.7\%, $p=0.699)$. On the other hand, $3(21.4 \%, p=0.404)$ of the IgG positive subjects were receiving steroids, $2(14.3 \%$, $p=1$ ) were receiving bDMARDs (adalimumab: 2 ), 2 $(14.3 \%, p=0.520)$ were receiving cDMARDs (azathioprine:1, methotrexate: 1 ), one was receiving colchicine $(7.1 \%, p=0.302)$, and one was receiving HCQ $(7.1 \%$, $p=0.467)$.

The parents of 5 of the $\operatorname{IgA}$ positive patients $(71.4 \%$, $p=0.633)$ and 4 of the $\operatorname{IgG}$ positive patients $(57.1 \%$, $p=0.155$ ) were thinking that their children were at increased risk for SARS-CoV-2 infection due to their chronic rheumatic diseases. Two of the IgA positive patients $(28.6 \%, p=0.108)$ and one of the $\operatorname{IgG}$ positive patients $(14.3 \%, p=0.473)$ had a poor drug compliance. It was noticed that neither $\operatorname{IgA}(p=0.195)$ positive nor $\operatorname{IgG}$ $(p=0.195)$ positive patients had attended their follow-ups regularly during the pandemic. Additionally, baseline data for each of the seropositive (IgA or IgG positive) subjects were given (Table 2).
Fig. 2 Antibody ratios of the subjects (FMF, familial Mediterranean fever group; $\mathrm{HC}$, healthy control group; Ig, immunoglobulin; JIA, juvenile idiopathic arthritis group; SLE, systemic lupus erythematosus group)
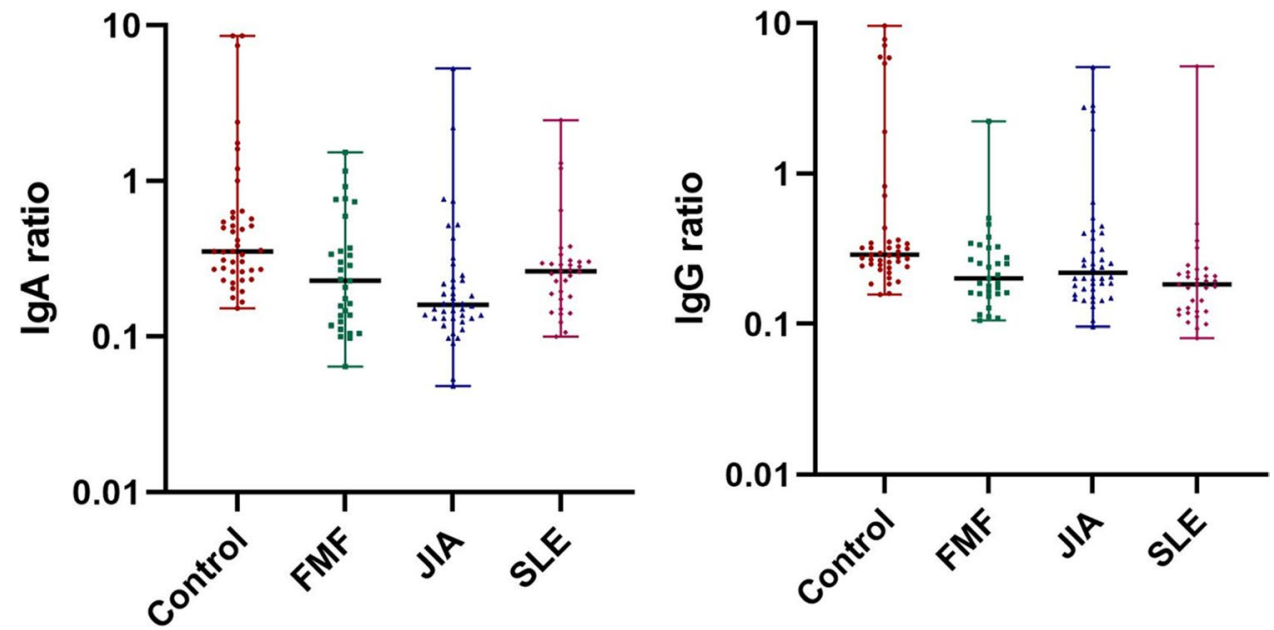


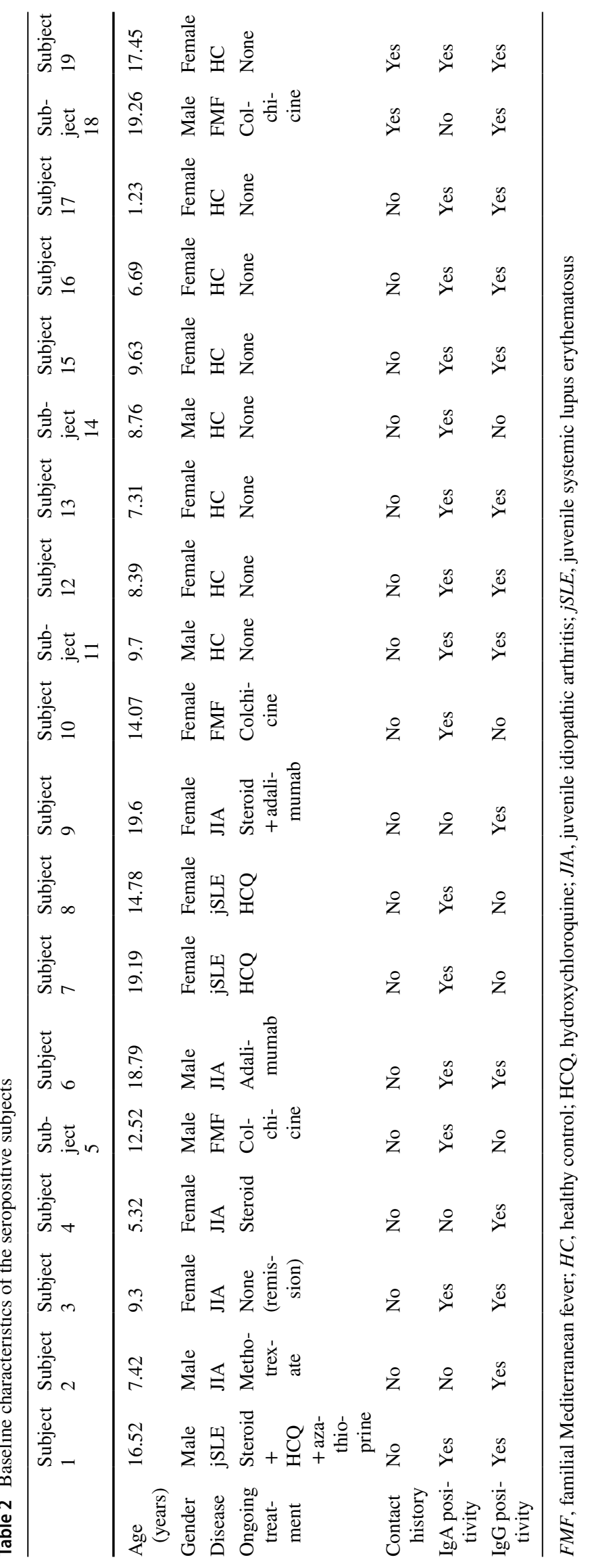




\section{Discussion}

In this study performed among 149 subjects, SARS-CoV-2 seropositivity was recorded in $19(12.75 \%)$ of them: antiSARS-CoV-2 IgA antibody in 15 (10\%) and anti-SARSCoV-2 IgG antibody in 14 (9.39\%) of them (HC: 8; JIA: 5; jSLE: 3; FMF: 3). Although not significant, seropositivity was more often in the HC group. Both IgA and IgG positivity were not found to be related to age, sex, underlying rheumatic diseases, and received treatments of the patients.

Although there are available data pointing the incidence and fatality rate of COVID-19, it must be considered that there are also unrecorded cases that can significantly change those data. Probably, most of the unrecorded cases are those that were not tested due to being asymptomatic. On the other hand, asymptomatic cases have a pivotal role in transmission. SARS-CoV-2 contagiousness of asymptomatic and symptomatic children was found to be similar [8]. Since the children are more likely to have asymptomatic disease course, clarifying whether they are hidden drivers of the pandemic is required. Furthermore, due to their immune-disturbed conditions caused by their diseases or treatments, the proportion of children with rheumatic diseases among these hidden transmitters is another conundrum.

It was recently mentioned in a comprehensive systematic review that the incidence of SARS-CoV-2 is $1.2-12.9 \%$ among asymptomatic individuals [14]. However, there is scarce data focused on the pediatric population. The incidence of SARS-CoV-2 among hospitalized asymptomatic children was found $1-2 \%$ by Paten et al. [15]. In a study from Belgium, eighty-four asymptomatic day-care children aged 6-30 months were tested, and although almost half of them had common cold symptoms, none of them was found positive. The main reason there were no positive patients was probably the fact that the study was conducted in the early days of the pandemic [16]. Anti-SARS-CoV-2 antibody measurements of 1109 children in Italy were analyzed, and it was reported that $67.8 \%$ of the seropositive subjects never had any suggestive symptom for COVID-19 [9]. However, there is no study evaluating the asymptomatic pediatric patients with rheumatic disease, so far. To our best knowledge, this is the first study focused on this group of patients.

Studies so far suggest that the disease is relatively rare and goes milder in children compared to adults [17]. A population screening from Iceland revealed that children under ten are less likely to be infected [18]. Six months later, in a seroprevalence study from China, it was demonstrated that anti-SARS-CoV-2 IgM antibody seropositivity was higher in younger children [19]. Hence, we speculate that even if the increasing age was considered a risk factor for being infected in the early days of the pandemic, it might be an illusion due to the limited social circumstances of younger individuals. However, since the risk for contacts with infected persons among children will increase with time, future population screening studies will be required.

It was demonstrated that SARS-CoV-2 enters into human cells via binding to angiotensin I-converting enzyme-2 (ACE-2), which serves as a receptor for the virus [20]. Indeed, while ACE- 2 catalyzes angiotensin I into angiotensin 1-9, an anti-inflammatory mediator, ACE turns the same substrate into angiotensin II, a pro-inflammatory mediator. Thus, downregulation of ACE- 2 by SARS-CoV-2 infection leads to a hyperinflammatory state [17]. Furthermore, it was recently demonstrated that ACE- 2 expression significantly decreases by aging [21]. Thus, even if younger patients are infected, they might have greater ACE-2, which has an antiinflammatory effect. These current findings make us consider that, while children are more likely to be infected, they are more likely to be asymptomatic, as well. However, the ages of the seropositive and seronegative subjects were not significantly different in our study. As it was well established previously, disease onsets of pediatric rheumatic conditions are mainly in school age and adolescence, and infants and toddlers are generally skipped [22]. Therefore, pediatric patients with rheumatic disease may not be a relevant population for estimating the age effect on being infected.

Although there was no significant difference between the groups, seropositivity was less frequent in patients with rheumatic diseases in the present study. Data so far suggest that infection rates are not higher in patients with rheumatic diseases, comparing to the general population [23]. Besides, it was shown that patients with rheumatic diseases had better compliance with isolation measurements than healthy individuals [24]. Consistently, in our study, only one patient with rheumatic diseases (one with FMF) while four subjects in the HC group had contact histories with a confirmed COVID-19 case. Thus, strict isolation measures taken by patients with rheumatic diseases may present a reasonable explanation for our results.

It is already known that patients with SLE are more susceptible to viral infections [25]. Besides, it was suggested that patients with SLE might be more prone to be infected and to have a severe COVID-19 disease course due to their inherent epigenetic dysregulations, regardless of their immunosuppressive treatment regimens [26]. However, SLE patients were not found to be at increased risk of infection and to have a severe disease course than the general population $[27,28]$.

In the early days of the pandemic, HCQ that is widely used in the treatment of SLE was reported to be quite safe and effective in the treatment of COVID-19 [29, 30]. In a short period of time, uncontrolled widespread clinical use 
of HCQ, not only for treatment but also for disease prevention, led to a shortage of HCQ among the patients who were under long-term treatment [31]. In April 2020, seventeen adult SLE patients with COVID-19 were reported. Their ongoing HCQ treatment was continued during the SARSCoV-2 infection. Eleven had respiratory failure, and two have died, unfortunately. However, most of them had comorbidities such as obesity and chronic kidney diseases [32]. A recently conducted study with a larger cohort reported a significantly lower SARS-CoV-2 infection rate among the patients chronically receiving HCQ, than general population. Therefore, it was suggested that the long-term HCQ treatment might protect against the infection [33]. On the contrary, no significant relationship was found between chronic use of HCQ and SARS-CoV-2 infection frequency in a cross-sectional multi-center study [34]. A population-based study evaluated the HCQ users for their SLE or rheumatoid arthritis, compared them with the general population, and found no evidence regarding the mortality-reducing effect of the HCQ prophylaxis [35]. Carbillon et al. [36] pointed out that comorbidities of the subjects in the studies should be meticulously evaluated before concluding that HCQ has no prevention effect on COVID-19. Consistently, three of our seropositive subjects were jSLE, none of them had obesity, hypertension, or chronic kidney diseases. All of them were teenagers, receiving HCQ and they were entirely asymptomatic, like the rest of the study population.

In one study among adults, 2 of 27 FMF patients with COVID-19 while in another study one of the 34 FMF patients with COVID-19 have died. However, they all had significant risk factors such as hypertension and obesity [37, 38]. No COVID-19-related death among pediatric patients with FMF has been reported yet. It was written in a previous study evaluating confirmed COVID-19 cases with childhood-onset FMF that all of the patients recovered completely, and severe complications were seen in none of them [3]. Consistently, among our asymptomatic seropositive subjects, three of them were FMF patients. All three had confirmed $M E F V$ gene mutations and were using colchicine during the pandemic. Although not examined regarding SARS-CoV-2 yet, it was previously suggested that $M E F V$ mutation might provide a biological advantage to the host for certain infectious agents such as Mycobacteria and Yersinia $[39,40]$. Moreover, although clinical trials are underway, favorable outcomes of colchicine in the treatment of COVID-19 were reported [41]. Thus, possible preventive effects of both MEFV mutation and colchicine merit to be further investigated.

Preliminary data suggest that either severity or frequency of COVID-19 is not increased in the patients with JIA [4]. It has been recently shown in a comprehensive study that neither JIA itself nor receiving b/cDMARDs was a significant risk factor for poor COVID-19-related outcomes [42]. Four
COVID-19 cases with JIA were reported in a recent paper, one was receiving HCQ, two were receiving methotrexate, and all of them were under anti-tumor necrosis factor (TNF) treatment. Anti-TNF treatments were discontinued in all of the patients, and none of them experienced severe clinical manifestations [43]. In our study, 5 of 19 seropositive subjects were diagnosed with JIA, two were receiving adalimumab, one was receiving methotrexate. Although DMARD medication was ceased in none of them, all of them were entirely asymptomatic.

Withdrawing DMARD medications, especially biologics, during pandemic due to their immunosuppressive effects is a debate. Indeed, each of the bDMARDs classes should be evaluated separately due to their different action mechanisms. Marques et al. [44] suggest that although receiving cyclophosphamide was associated with poor outcomes; long-term anti-TNF medication may provide protection against the COVID-19. In a recent adult study, while rituximab treatment was associated with higher hospitalization and death rates, anti-TNF treatment was not found as a risk factor for severe COVID-19 [45]. Consistently, two of our asymptomatic COVID-19 subjects were under an anti-TNF agent treatment named adalimumab.

We conducted the present study by using EUROIMMUN commercial kits for anti-SARS-CoV-2 antibodies. It was previously shown that the overall specificities of $\operatorname{IgA}$ and IgG were $94.7 \%$ and $97.1 \%$, respectively [46]. Therefore, positive results in this study were considered highly suggestive for the novel coronavirus infection. Considering the pivotal role of the IgA on mucosal immunity, while clinicians are mainly focused on IgM and IgG, we chose to evaluate the IgA instead of IgM ratios. Consistently, it was speculated in a study by Zhang et al. [47] that IgA-based ELISA kit is a more sensitive tool than IgM and IgG, for diagnosing acute COVID-19. Besides, we aimed to perform the long-term monitorization of antibody levels in seropositive subjects in future studies. Therefore, for estimating seropositivity, we preferred the semi-quantitative method instead of qualitative method.

The main limitation of our study is the relatively small number of subjects for estimating the seroprevalence, which is mainly due to the limited count of obtained commercial kits because of the economic burden of antibody testing. Additionally, up to date, there has been no data regarding the frequency of asymptomatic seropositivity among neither healthy children nor children with rheumatic diseases. Therefore, we were not able to calculate the optimal sample size. Another limitation is that we did not assess the diseases activities of the patients.

In conclusion, we conducted a cross-sectional study evaluating the serological status of pediatric patients with rheumatic diseases for asymptomatic SARS-CoV-2 infection. In this study, there was no significant difference between 
healthy children and patients with childhood-onset rheumatic diseases regarding the asymptomatic SARS-CoV-2 seropositivity. We revealed that patients with childhoodonset rheumatic diseases, even if they receive immunosuppressive medication such as bDMARDs or cDMARDs, might have an asymptomatic SARS-CoV-2 infection, similarly to their healthy peers. Further studies with larger sample sizes are required to verify these promising results.

Author contribution FH, DO, MY, A Adrovic, SS, O Koker, A Aliyeva, VG, GY, GI, BK, O Kasapcopur, KB were responsible for data collection and analysis. FH, DO, MY, A Adrovic, SS contributed to the writing of the manuscript. KB, O Kasapcopur, KB reviewed and revised the manuscript.

Funding This work was supported by the Scientific Research Projects Coordination Unit of Istanbul University-Cerrahpasa. ID: 34942, Project code: TSA-2020-34942.

Data sharing statement All data relevant to the study are included in the article.

\section{Declarations}

Ethics approval The study was approved by the Institutional Review Board of our University (04/16/20-29430533-604.01-01-54959).

\section{Disclosures None.}

Informed consent A written informed consent was obtained from all the participants included in this study and no identifying information of any participant was included in this paper.

The patient and public involvement statement Patients or the public were not involved in the design, or conduct, or reporting, or dissemination plans of our research.

\section{References}

1. Ramírez ML, Martinez SM, Bessone CDV, Allemandi DA, Quinteros DA (2021) COVID-19: epidemiological situation of Argentina and its neighbor countries after three months of pandemic. Disaster Med Public Health Prep 25:1-7

2. Ludvigsson JF (2020) Systematic review of COVID-19 in children shows milder cases and a better prognosis than adults. Acta Paediatr 109(6):1088-1095

3. Haslak F, Yildiz M, Adrovic A, Sahin S, Koker O, Aliyeva A et al (2020) Management of childhood-onset autoinflammatory diseases during the COVID-19 pandemic. Rheumatol Int 40(9):1423-1431

4. Yildiz M, Haslak F, Adrovic A, Sahin S, Barut K, Kasapcopur O (2020) The frequency and clinical course of COVID-19 infection in children with juvenile idiopathic arthritis. Clin Exp Rheumatol 38(6):1271-1272

5. Monti S, Balduzzi S, Delvino P, Bellis E, Quadrelli VS, Montecucco C (2020) Clinical course of COVID-19 in a series of patients with chronic arthritis treated with immunosuppressive targeted therapies. Ann Rheum Dis 79(5):667-668
6. Ouédraogo DD, Tiendrébéogo WJS, Kaboré F, Ntsiba H (2020) COVID-19, chronic inflammatory rheumatic disease and antirheumatic treatments. Clin Rheumatol 39(7):2069-2075

7. Nikolai LA, Meyer CG, Kremsner PG, Velavan TP (2020) Asymptomatic SARS coronavirus 2 infection: invisible yet invincible. Int J Infect Dis 100:112-116

8. Cai J, Wang X, Zhao J, Ge Y, Xu J, Tian H et al (2020) Comparison of clinical and epidemiological characteristics of asymptomatic and symptomatic SARS-CoV-2 infection in children. Virol Sin 35(6):803-810

9. Barcellini L, Forlanini F, Sangiorgio A, Gambacorta G, Alberti L, Meta A, et al. (2021) Does school reopening affect SARS-CoV-2 seroprevalence among school-age children in Milan? PLoS One 16(9):e0257046.

10. Petri M, Orbai AM, Alarcón GS, Gordon C, Merrill JT, Fortin PR et al (2012) Derivation and validation of the Systemic Lupus International Collaborating Clinics classification criteria for systemic lupus erythematosus. Arthritis Rheum 64(8):2677-2686

11. Yalçinkaya F, Ozen S, Ozçakar ZB, Aktay N, Cakar N, Düzova A et al (2009) A new set of criteria for the diagnosis of familial Mediterranean fever in childhood. Rheumatology (Oxford) 48(4):395-398

12. Petty RE, Southwood TR, Manners P, Baum J, Glass DN, Goldenberg J et al (2004) International League of Associations for Rheumatology classification of juvenile idiopathic arthritis: second revision, Edmonton, 2001. J Rheumatol 31(2):390-392

13. Lauer SA, Grantz KH, Bi Q, Jones FK, Zheng Q, Meredith HR et al (2020) The incubation period of coronavirus disease 2019 (COVID-19) from publicly reported confirmed cases: estimation and application. Ann Intern Med 172(9):577-582

14. Al-Sadeq DW, Nasrallah GK (2020) The incidence of the novel coronavirus SARS-CoV-2 among asymptomatic patients: a systematic review. J Infect Dis 98:372-380

15. Patel AB, Clifford A, Creaden J, Kato K, Malakooti MR, Muller WJ et al (2020) Severe acute respiratory syndrome coronavirus 2 point prevalence among asymptomatic hospitalized children and subsequent healthcare worker evaluation. J Pediatric Infect Dis Soc 9(5):617-619

16. Desmet S, Ekinci E, Wouters I, Decru B, Beuselinck K, Malhotra-Kumar S et al (2020) No SARS-CoV-2 carriage observed in children attending daycare centers during the intial weeks of the epidemic in Belgium. J Med Virol 93(3):1828-1831

17. Haşlak F, Yıldız M, Adrovic A, Barut K, Kasapçopur Ö (2020) Childhood rheumatic diseases and COVID-19 pandemic: an intriguing linkage and a new horizon. Balkan Med J 37(4):184-188

18. Gudbjartsson DF, Helgason A, Jonsson H, Magnusson OT, Melsted P, Norddahl GL et al (2020) Spread of SARS-CoV-2 in the Icelandic population. N Engl J Med 382(24):2302-2315

19. Wang R, Jin F, Cao S, Yuan H, Qu J, Zhang J et al (2020) Seroprevalence of SARS-CoV-2 infections among children visiting a hospital. Pediatr Investig 4(4):236-241

20. Zhou P, Yang XL, Wang XG, Hu B, Zhang L, Zhang W et al (2020) A pneumonia outbreak associated with a new coronavirus of probable bat origin. Nature 579(7798):270-273

21. Chen J, Jiang Q, Xia X, Liu K, Yu Z, Tao W, et al. (2020) Individual variation of the SARS-CoV-2 receptor ACE2 gene expression and regulation. Aging cell 19(7).

22. Huemer C, Huemer M, Dorner T, Falger J, Schacherl H, Bernecker $\mathrm{M}$ et al (2001) Incidence of pediatric rheumatic diseases in a regional population in Austria. J Rheumatol 28(9):2116-2119

23. Michelena $\mathrm{X}$, Borrell $\mathrm{H}$, López-Corbeto M, López-Lasanta M, Moreno E, Pascual-Pastor M et al (2020) Incidence of COVID-19 in a cohort of adult and paediatric patients with rheumatic diseases treated with targeted biologic and synthetic disease-modifying anti-rheumatic drugs. Semin Arthritis Rheum 50(4):564-570 
24. Hooijberg F, Boekel L, Vogelzang EH, Leeuw M, Boers M, van Vollenhoven R et al (2020) Patients with rheumatic diseases adhere to COVID-19 isolation measures more strictly than the general population. Lancet Rheumatol 2(10):e583-e585

25. Katsuyama E, Suarez-Fueyo A, Bradley SJ, Mizui M, Marin AV, Mulki L et al (2020) The CD38/NAD/SIRTUIN1/EZH2 axis mitigates cytotoxic CD8 $\mathrm{T}$ cell function and identifies patients with SLE prone to infections. Cell Rep 30(1):112-23.e4

26. Sawalha AH, Zhao M, Coit P, Lu Q (2020) Epigenetic dysregulation of ACE2 and interferon-regulated genes might suggest increased COVID-19 susceptibility and severity in lupus patients. Clin Immunol 215:108410.

27. Mason A, Rose E, Edwards CJ (2020) Clinical management of Lupus patients during the COVID-19 pandemic. Lupus 29(13):1661-1672

28. Favalli EG, Gerosa M, Murgo A, Caporali R (2021) Are patients with systemic lupus erythematosus at increased risk for COVID19? Ann Rheum Dis 80(2):e25.

29. Venerito V, Lopalco G, Iannone F (2020) COVID-19, rheumatic diseases and immunosuppressive drugs: an appeal for medication adherence. Rheumatol Int 40(5):827-828

30. Gautret P, Lagier J-C, Parola P, Meddeb L, Mailhe M, Doudier B, et al. (2020) Hydroxychloroquine and azithromycin as a treatment of COVID-19: results of an open-label non-randomized clinical trial. Int J Antimicrob Agents 57(1):106243

31. Kim AH, Sparks JA, Liew JW, Putman MS, Berenbaum F, Duarte-García A et al (2020) A rush to judgment? Rapid reporting and dissemination of results and its consequences regarding the use of hydroxychloroquine for COVID-19. Ann Intern Med 172(12):819-821

32. Mathian A, Mahevas M, Rohmer J, Roumier M, Cohen-Aubart F, Amador-Borrero B et al (2020) Clinical course of coronavirus disease 2019 (COVID-19) in a series of 17 patients with systemic lupus erythematosus under long-term treatment with hydroxychloroquine. Ann Rheum Dis 79(6):837-839

33. Ferreira A, Oliveira ESA, Bettencourt P (2021) Chronic treatment with hydroxychloroquine and SARS-CoV-2 infection. J Med Virol 93(2):755-759

34. Tejada Cifuentes F, Lloret Callejo Á, Tirado Peláez MJ, Rubio Pulido O, Ruiz-Morote Aragón M, Fernández Urrusuno R et al (2021) Incidence of COVID-19 in patients under chronic treatment with hydroxychloroquine. Med Clin (Engl Ed) 156(4):166-171

35. Rentsch CT, DeVito NJ, MacKenna B, Morton CE, Bhaskaran K, Brown JP et al (2021) Effect of pre-exposure use of hydroxychloroquine on COVID-19 mortality: a population-based cohort study in patients with rheumatoid arthritis or systemic lupus erythematosus using the OpenSAFELY platform. Lancet Rheumatol 3(1):e19-e27

36. Carbillon L, Benbara A, Boujenah J (2020) Clinical course of COVID-19 in patients with systemic lupus erythematosus under long-term treatment with hydroxychloroquine. Ann Rheum Dis 80: e54.
37. Bourguiba R, Delplanque M, Vinit C, Ackermann F, Savey L, Grateau G, et al. (2020) Clinical course of COVID-19 in a cohort of 342 familial Mediterranean fever patients with a long-term treatment by colchicine in a French endemic area. Ann Rheum Dis; annrheumdis-2020-218707

38. Güven SC, Erden A, Karakaş Ö, Armağan B, Usul E, Omma A et al (2021) COVID-19 outcomes in patients with familial Mediterranean fever: a retrospective cohort study. Rheumatol Int 41(4):715-719

39. Cattan D (2005) MEFV mutation carriers and diseases other than familial Mediterranean fever: proved and non-proved associations; putative biological advantage. Curr Drug Targets Inflamm Allergy 4(1):105-112

40. Chung LK, Park YH, Zheng Y, Brodsky IE, Hearing P, Kastner DL et al (2016) The Yersinia virulence factor YopM hijacks host kinases to inhibit type III effector-triggered activation of the pyrin inflammasome. Cell Host Microbe 20(3):296-306

41. Della-Torre E, Della-Torre F, Kusanovic M, Scotti R, Ramirez GA, Dagna L, et al. (2020) Treating COVID-19 with colchicine in community healthcare setting. Clin Immunol 217:108490.

42. Bower H, Frisell T, Di Giuseppe D, Delcoigne B, Ahlenius GM, Baecklund $E$ et al (2021) Impact of the COVID-19 pandemic on morbidity and mortality in patients with inflammatory joint diseases and in the general population: a nationwide Swedish cohort study. Ann Rheum Dis 80(8):1086-1093

43. Marino A, Romano M, Gattinara M, Cimaz R (2020) Patients with juvenile idiopathic arthritis on TNF inhibitors exposed to COVID19 family members. Semin Arthritis Rheum 50(6):1214-1215

44. Marques CDL, Kakehasi AM, Pinheiro MM, Mota LMH, Albuquerque CP, Silva CR, et al. (2021) High levels of immunosuppression are related to unfavourable outcomes in hospitalised patients with rheumatic diseases and COVID-19: first results of ReumaCoV Brasil registry. RMD Open 7(1): e001461

45. Bachiller-Corral J, Boteanu A, Garcia-Villanueva MJ, de la Puente C, Revenga M, Diaz-Miguel MC et al (2021) Risk of severe coronavirus infection (COVID-19) in patients with inflammatory rheumatic diseases. J Rheumatol 48(7):1098-1102

46. Kowitdamrong E, Puthanakit T, Jantarabenjakul W, Prompetchara E, Suchartlikitwong P, Putcharoen O, et al. (2020) Antibody responses to SARS-CoV-2 in patients with differing severities of coronavirus disease 2019. PloS one 15(10):e0240502.

47. Zhang C, Lin L, Tang D, Liu F, Li M, Li Q et al (2021) Antibody responses to SARS-CoV-2 in healthy individuals returning to Shenzhen. J Med Virol 93(2):1154-1157

Publisher's Note Springer Nature remains neutral with regard to jurisdictional claims in published maps and institutional affiliations.

\section{Authors and Affiliations}

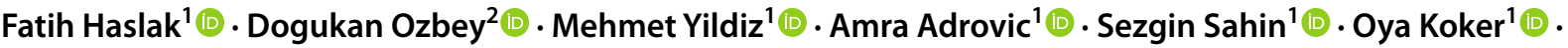

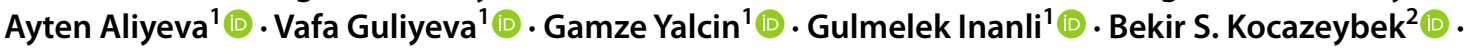 Ozgur Kasapcopur ${ }^{1}\left[\right.$ (D) Kenan Barut ${ }^{1}$ (1)}

Fatih Haslak

drfatihhaslak@gmail.com

Dogukan Ozbey

dogukanozbey@gmail.com
Mehmet Yildiz

yildizmehmet@istanbul.edu.tr

Amra Adrovic

amra.adrovic@istanbul.edu.tr 
Sezgin Sahin

sezgin@istanbul.edu.tr

Oya Koker

oyakoker@hotmail.com

Ayten Aliyeva

aesa099@mail.ru

Vafa Guliyeva

doktor_guliyeva@hotmail.com

Gamze Yalcin

gmzeylcnn@gmail.com

Gulmelek Inanli

gulmelek.inanli@gmail.com
Bekir S. Kocazeybek

bzeybek@istanbul.edu.tr

Ozgur Kasapcopur

ozgurkasapcopur@hotmail.com

1 Department of Pediatric Rheumatology, Istanbul UniversityCerrahpasa Cerrahpasa Medical School, Istanbul, Turkey

2 Department of Microbiology, Istanbul University-Cerrahpasa Cerrahpasa Medical School, Istanbul, Turkey 